\title{
Variations from the Prototypic Near-Death Experience: The "Individually Tailored" Hypothesis
}

\author{
William J. Serdahely, Ph.D. \\ Montana State University
}

ABSTRACT. A nonrandom sample of firsthand accounts of near-death experience (NDEs) revealed a number of variations from the prototypic NDE description, including feeling judged during a life review, seeing a nondeceased friend in the tunnel, experiencing no pain upon returning to the physical body, and crossing a barrier before being sent back. The prototypic NDE model is useful in giving an overview of NDEs, but ultimately the theoretical framework of near-death studies will have to accommodate these and other variations from the prototype. The "individually tailored" hypothesis, put forth to account for many of these variations, states that each NDEr receives what he or she needs during the NDE in a way that NDEr is able to accept.

A description of the prototypic near-death experience (NDE) frequently appears in the literature. For example, Cherie Sutherland wrote $(1992$, p. 3):

The near-death episode itself is typically characterised by a feeling of peace, an out-of-body experience, the sensation of travelling very quickly through a dark tunnel, generally towards a light, an en. counter with the spirits of deceased relatives or friends or a 'Being of Light,' an instantaneous life-review, and for some, entrance into a world of light.

Sutherland's prototype is similar to the one proposed by Kenneth Ring (1980). Margot Grey's comparative study of near-death experi-

William J. Serdahely, Ph.D., is Professor of Health Science at Montana State University. Reprint requests should be addressed to Dr. Serdahely at the Department of Health and Human Development, Montana State University, Bozeman, MT 59717. 
ences in the United Kingdom yielded a model that is also similar to the 5-stage model presented by Ring in 1980 . Grey wrote $(1985, \mathrm{p}$. 32): “. . . in general I think the model I adopted basically resembles the one used by Dr. Ring."

Ring himself noted in his second NDE book, Heading Toward Omega (1984), that the narration of the typical NDE given in his earlier book, Life at Death (1980), on the whole "holds up very well" (1984, p. 38). He credited Raymond Moody with publishing the earliest version of this prototypic NDE in Life After Life (1975), in which Moody had written (pp. 21-22) that the NDEr

\begin{abstract}
begins to hear an uncomfortable noise, a loud ringing or buzzing, and at the same time feels himself moving very rapidly through a long dark tunnel. ... [A] being of light [helps] him evaluate his life .. . by showing him a panoramic, instantaneous playback of the major events of his life. At some point he finds himself approaching some sort of barrier or border, apparently representing the limit between earthly life and the next life. ... He is overwhelmed by in. tense feelings of joy, love, and peace ... [and] somehow reunites with his physical body.
\end{abstract}

The prototypic description of an NDE can also be found commonly in the popular literature and on television programs devoted to near-death experiences. In the many NDE talks I have given, I have invariably presented the same pattern described by the above authors.

Yet as I and all of the above reseachers have noted, many variations from this model can be found. And it is to these variations that the remainder of this paper will be devoted, in an effort to expand the model to accommodate more completely events described to us by NDErs.

NDE findings, especially veridical out-of-body experiences (Ring and Lawrence, 1993), present the world of science with anomalous data, data that cannot be accommodated easily by its present theories. Near-death researchers have "held up" these anomalous findings for the scrutiny of both the scientific and medical communities. In the same tradition, "holding up" variations from the prototypic NDE allows us to continue the paradigm shift undertaken since the publication of Life After Life (Moody, 1975), and save us from practicing what the historian of science Thomas Kuhn (1962) called "normal 
science." That is to say, it prevents us from being limited by the parameters established by the prototypic NDE model. It does not allow the prototype to blind us to the myriad variations from the NDE model.

As Ring cautioned, "This prototypical summary creates the risk of some distortion and idealization of the experience" (1980, p. 101). By focusing on the variations from the prototype, the attempt here is to minimize the distortion and to build a model that is more congruent with all of the NDE reportings.

\section{A Nonrandom Sample}

During my recent sabbatical I encountered 11 people who had had an NDE and one who had had a series of six out-of-body experiences (OBEs) following a traumatic incident. Six of these experiencers were female and six were male.

In addition to these 12 people, I also heard secondhand the NDEs of three more individuals. One of these NDErs had recently died. Her husband and adult daughter attended my NDE class and told me about the two NDEs the woman had had, one at age 9 and the other at about age 47. Another secondhand report came from the grandmother and the mother of a girl who was born 3 months prematurely and was almost 4 years old at the time I met with the grandmother. The third secondhand report came from one of the NDErs in my nonrandom sample, who said that while he was hospitalized at a time concurrent with his own NDE, a man two beds away from him told him of a "hellish" NDE he had just gone through. The first man had asked the second why he had been screaming a short time before, and was then told about the hell-like NDE.

The age range of the firsthand reporters at the time of their NDEs was from about age 5 or 6 to age 60 . Melissa, who was born 3 months prematurely, apparently had her NDE while she was still in the incubator following her premature birth; this case is similar to the one reported previously by Barbara Walker and me, in which our respondent believed she had had an NDE at birth (Serdahely and Walker, 1990). 


\section{Variations from the Prototype}

\section{Distressing NDEs}

Four of these individuals experienced their NDEs as unpleasant. Three of them said it was "frightening," "scary," and "unpleasant," and that they experienced fear or were "afraid" during their NDE. One of the secondhand reports described a 9 year old girl being frightened when she went downward in a dark, black tunnel to a golden, loving light. None of these four NDErs described any "hellish" imagery.

These four cases fit the most common category of distressing NDE as outlined by Bruce Greyson and Nancy Evans Bush (1992). Presumably, all four were having a version of the prototypic near-death experience as described by Sutherland above. But instead of finding it pleasant and peaceful, they found it frightening and scary. In the secondhand account, because the 9 year old girl was traveling down. ward and had been taught that hell was down, she feared she was going to hell, although she was said to have later seen a golden, loving light as her NDE continued to unfold.

Unlike the above four NDErs, two persons in my nonrandom sam. ple experienced hellish imagery. One of the six OBEs of a woman respondent was hell-like with "ugly gargoyles" and the "devil." (This same woman had a subsequent OBE that filled her with love and peace.)

A second person from my sample of secondhand accounts was said to have changed after his hellish NDE from being a "jerk" to becom. ing a "nice guy." While the transformative nature of a hellish NDE was alluded to by Maurice Rawlings (1993), it was never as fully nor as clearly explicated as it could be.

\section{Skipping a Stage}

Four NDErs had no recollection of the OBE stage, the second stage in the typology of Ring (1980) and Grey (1985). They reported they went immediately into a tunnel on the way to the light.

Two had no recollection of a tunnel, the third stage in Ring's and Grey's typology, even though they had a light experience. They found themselves out of their bodies viewing the scene below them and then proceeded to have a light experience. One of these two persons 
had a flashforward during her light experience; the other, who had multiple OBEs, went from the out-of-body stage into the light on at least three occasions.

\section{Tunnels}

A number of variations from the prototypic tunnel were reported by my nonrandom sample. One person experienced a "clockwise spinning vortex" of blacks, whites, and grays; one reported an "all gray whirlpool"; one said the tunnel was like a "windsock," like a "slinky covered with nylon"; another said the tunnel was not solid but "netlike," like a "spider web"; and still another reported many side tunnels coming off the main tunnel, through which he and his two deceased grandfathers were traveling. Deceased people came out of the side tunnels to greet this NDEr.

One person went through a mist or a fog (and not a dark void or tunnel) to get to the light, and another passed through the hospital wall into a fog or into a gray, cloudy mist before encountering the main tunnel with the multiple side tunnels.

One of the female NDErs saw a living female friend in her "windsock" tunnel. The friend told her to go back to her body. It is rare to hear of a living friend or relative appearing during a near-death experience. However, the gender of the comforting spirit is consistent with previous reports of females appearing to women who are victims of sexual abuse and of sexual assault, as this woman was (Serdahely, 1987-88, 1992, 1993).

One male respondent said he traveled through a tunnel on a cart or gurney on his way to being greeted by his deceased father, godfather, and coworkers, all of whom beckoned him into the light. I had previously come across an NDEr who indicated he had been transported to the light in an ambulance-like vehicle.

\section{Life Review}

Three respondents reported having a life review. The form of these life reviews varied from the prototypic description of a panoramic view with concurrent feelings of empathy in the company of a "being of light." 
One person saw portions of his life in "five-minute intervals." An. other viewed only the significant events of his life with the exclusion of any "mundane recollections." His life review happened at the very beginning of his NDE, as he was drowning at age 12, whereas life reviews are more commonly reported later in the NDE, in the tunnel or in the world of light.

The woman with multiple OBEs said her life review was like watching prints from a camera. All of the highlights were in chronological order. However, some of the prints were blank, and she was informed that these blank ones represented her future.

A major variation from the prototypic NDE came with the life review of the first NDEr above, who saw his life in "five-minute intervals." This person said he felt judged during his life review. The judgment came from four beings of light who were positioned above him, plus an additional being who was in the background or in another room. The common report is that no judgment is felt during the life review. This NDEr also said he was given praise during the review and felt the life review was an opportunity for him to learn from his mistakes.

\section{Music}

Four persons heard music or sounds of an ineffable quality. The NDEr who felt judged during his life review heard soft, beautiful, symphonic music later in his near-death experience. Another heard angelic voices. They were not singing words but notes of various modulations and frequencies. The music was melodious, comforting, and soothing.

One woman heard "unearthly" music from which love emanated. The music drew her into the world of light and was sung by a choir. She said that if she had gone into the music, she would not have returned to her body. A fourth NDEr did not hear music as such but a background sound that he said was quite pleasureable.

\section{Messages Given During the NDE}

While receiving messages during an NDE is not in itself unusual, three people in this nonrandom sample received unique messages. 
The woman whose NDE resulted from a sexual assault was told that it was "all right" (that is, safe) for her to go back to her body.

The man who discovered side tunnels coming off the main tunnel was informed by a deceased grandfather that he needed to return to father two sons. He reported that he and his wife did have two sons after his NDE. And the NDEr who experienced the flashforward, at another point in her NDE, felt a higher power wanted her to have a second child.

\section{Barriers or Being Told to Return}

Being told to go back to one's physical body is not an unusual finding in itself. Three of these respondents were so told. One of the three was instructed apparently by a deceased grandmother not to cross a line in front of her. The OBEr did cross the line, at which point the grandmother said, "I told you not to cross the line." The older woman "got right in [her] face" and said, "You are to go back now!" This case is reminiscent of that of Tom Sawyer, who said he had been "kicked out of Heaven" (Ring, 1984).

Other respondents encountered barriers besides the above described barrier represented by a line. The woman who was attacked found the distal end of the tunnel blocked by a male figure she identified as her deceased father, whose presence created a barrier to moving into the world of light. And the man who was transported by a cart or gurney through the tunnel found two "concrete doors" closing on the world of light before he could enter it and join his father, godfather, and coworkers.

Finally, it is possible that the unpleasant affective tone of the three NDErs, who were minors at the time of their frightening NDEs (ages 5 or 6,8 , and 12 respectively), served as a barrier to further passage down the tunnel. The frightening, unpleasant quality apparently prevented them from going on and, in effect, sent them back to their bodies.

\section{Pain on Returning to the Physical Body}

The usual scenario is for NDErs to be pain-free once they have separated from their bodies, even though they may have been in excruciating pain prior to their NDE, and for the pain to return 
once they are back in the body. One of my respondents, who was in intense pain during the delivery of her first child, said she experienced no pain upon returning to the body. Prior to having her OBE, she said the pain was "extreme," "tremendous," and "excruciating." After the delivery she said she could see the physician stitching her up but felt no pain from the stitching. Her doctor asked her if she wanted a local anesthetic, but she refused, indicating she did not need it.

\section{Aftereffects}

The near-death literature over the past 18 years has described a number of commonly reported aftereffects (Hoffman, 1992; Moody and Perry, 1988; Sutherland, 1992). The people in my nonrandom sample also experienced some of these same aftereffects.

Several respondents said they felt more compassionate now. Two believed they had become more psychic. One indicated her OBEs, especially the one with the life review, alleviated her suicidal idea. tion. And one person reported having premonitions as a sequela to his NDE.

However, two of my respondents noted atypical aftereffects. One said that after his mother-in-law died, he had a strong sense that he needed to take care of the funeral arrangements for her, which he did. Two weeks after the funeral, he had an apparition of his mother-in-law and her deceased mother. The former thanked him for handling the matters related to the funeral, and the latter said he had been selected for this task because he "had already been among us," that is, among the dead during his NDE. This was the NDEr who had been greeted by deceased persons coming out of the side tunnels as he traveled through the main tunnel with his two deceased grandfathers.

Another respondent also had an unusual aftereffect. After her NDE associated with the difficult delivery of her first child, she had the sense that her higher power wanted her to have a second child, which she did. She, of course, was concerned that the second delivery would be as difficult as the first, but it was not. She said she felt the sense of having a second child may have been a "test of [her] faith." 


\section{Analysis}

Each of the 11 firsthand NDE reports was analyzed in the following way. Using Bruce Greyson's (1983) four components of the NDE (Cognitive, Affective, Paranormal, and Transcendental), all 11 NDEs were scored for each of the four components. A zero was assigned if the component was absent; a score of 1 was given if the component was present but of a low magnitude or not very prominent in the overall NDE; and a rating of 2 was awarded if the component was a very prominent part of the NDE or of a high magnitude. The respondents in this nonrandom sample were not given Greyson's 16-item questionnaire in part because the scale would not have picked up the unpleasant affective quality reported by three of the respondents nor the various tunnel experiences described above.

Using this rating system, each NDE could be described by a series of 4 numbers. For example, the experience of the woman who, when assaulted, went out of her body into a "windsock" tunnel and then encountered a living female friend would be scored as Cognitive = 1 , Affective $=1$, Paranormal $=2$, Transcendental $=0$. The NDE of the man who was judged during his life review was scored as Cognitive $=2$, Affective $=0$, Paranormal $=0$, Transcendental $=1$. The woman who at age 5 or 6 felt very frightened by being in a vortex before returning to her body was scored as Cognitive $=0$, Affective $=2$, Paranormal $=2$, Transcendental $=0$.

While there are some obvious methodological flaws with analyzing the data in this fashion, nevertheless the analysis does demonstrate the unique and individual nature of these NDEs. Most of the NDEs in this nonrandom sample varied in their mixture and magnitude of the four components, and even those NDEs that received identical ratings had variations from each other that were not picked up by this rating system. For example, for two NDErs with identical ratings on all four components, one went into a vortex while the other remained stationary at its entrance.

Clearly, the NDEs within this nonrandom sample differ from one another and, in a number of instances, vary greatly from many of the other NDEs reported previously. 


\section{The "Individually Tailored" Hypothesis}

One hypothesis to account for the many variations from the prototype is that each experiencer gets what he or she needs during the NDE in a way that he or she can accept it. The NDE or OBE is tailored specifically to fit the needs of that person. For example, the woman who was sexually assaulted was able to dissociate from the trauma by having an out-of-body experience. The friend she encountered in her experience was a "big woman" who worked for the sheriff's department that had jurisdiction for the county in which the assault took place and appeared to the NDEr in her sheriff's uniform.

If the NDE or OBE is to be a learning experience, it must be configured in a way that best teaches the person what he or she needs to know. The prominent feature of one NDE in this nonrandom sam. ple was the life review in which the individual felt judged. The judgment seems to have taught this NDEr what he needed to learn from mistakes he had previously made; his wife confirmed that he was a more positive person after his NDE. It may not have been spurious that he experienced neither a separation from his physical body nor a tunnel.

It should be noted that the "individually tailored" hypothesis is not reductionistic. It is not my assumption here that the specific alteration of the experience is created by the experiencer's brain. Second, the hypothesis is being proposed only for those NDEs and OBEs that happen spontaneously. The "individually tailored" hypothesis is not put forth to account for instances in which, for example, some people appear to leave their bodies at will and travel during the out-of-body state.

Approximately 60 percent of those who come close to death do not report having had an NDE (Ring, 1984). How are we to explain this percentage? Perhaps some of these 60 percent do not choose to reveal they have had an NDE, and perhaps some may be people who have medication-induced amnesia for their experiences. But perhaps some of those 60 percent may be people who, for whatever reason, do not need an NDE. For example, they may not need a "spiritual awak. ening" because of the spiritual work they have already done or the non-death-related transpersonal crises they have already endured.

Each person in this nonrandom sample received an experience that was tailored to his or her individual needs. Only one person had an experience that closely resembled the prototypic one. The people in this sample got what they needed in a way they could accept it. Hav. 
ing the prototypic experience may not have provided them with whatever they needed at the time, whether it was in terms of experiencing the safety of a friend, the learning gained from being judged in a life review, or the need to return to one's body due to being frightened by the experience.

The "individually tailored" hypothesis accounts for a great deal of the variation in the near-death experiences in this nonrandom sample. It helps to explain the variation in tunnels, the skipping of stages, the command to return or the option to stay, as well as many of the other variations discussed above.

\section{Conclusion}

The model of the prototypic NDE is helpful in giving an overview of the pattern that seems to transcend not only cultures but also historical time. However, as the above nonrandom sample demonstrates, many fascinating variations from, and remarkable exceptions to, the classic NDE model are reported by NDErs.

As near-death studies continues to grow, the variations from the model will need to be accounted for by the theoretical framework explaining NDEs. Thomas Kuhn pointed out over thirty years ago that not to take into consideration the anomalous findings is to practice "normal science," that is, "business as usual" within that established discipline (Kuhn, 1962).

The prototypic model can be modified by the "individually tailored" hypothesis, which states that when an NDE or OBE happens spontaneously, the experiencer receives whatever he or she needs at that time in a way that he or she can accept. We can then expect to find some mixture or combination of Greyson's four components for each NDE. The weighting of each component for a given NDE will depend on the needs of the experiencer at the time he or she is having that near-death experience.

\section{References}

Grey, M. (1985). Return from death: An exploration of the near-death experience. London, England: Arkana.

Greyson, B. (1983). The Near-Death Experience Scale: Construction, reliability, and validity. Journal of Nervous and Mental Disease, 171, 369.375. 
Greyson, B., and Bush, N. E. (1992). Distressing near-death experiences. Psychiatry, $55,95-110$.

Hoffman, E. (1992). Visions of innocence: Spiritual and inspirational experiences of childhood. Boston, MA: Shambhala.

Kuhn, T. (1962). The structure of scientific revolutions. Chicago, IL: University of Chicago Press.

Moody, R. A., Jr. (1975). Life after life. Covington, GA: Mockingbird Books.

Moody, R. A., Jr., and Perry, P. (1988). The light beyond. New York, NY: Bantam.

Rawlings, M. (1993). To hell and back. Nashville, TN: Thomas Nelson.

Ring, K. (1980). Life at death: A scientific investigation of the near-death experience. New York, NY: Coward, McCann and Geoghegan.

Ring, K. (1984). Heading toward omega: In search of the meaning of the near-death experience. New York, NY: William Morrow.

Ring, K., and Lawrence, M. (1993). Further evidence for veridical perception during near-death experiences. Journal of Near-Death Studies, 11, 223-229.

Serdahely, W. J. (1987.88). The near-death experience: Is the presence always the higher self? Omega, 18, 129-134.

Serdahely, W. J. (1992). Similarities between near-death experiences and multiple personality disorder. Journal of Near-Death Studies, 11, 19-38.

Serdahely, W. J. (1993). Near-death experiences and dissociation: Two cases. Journal of Near-Death Studies, 12, 85-94.

Serdahely, W. J., and Walker, B. A. (1990). A near-death experience at birth. Death Studies, 14, 177.183.

Sutherland, C. (1992). Transformed by the light: Life after near-death experiences. Sydney, Australia: Bantam. 